\title{
Symmetry energy at subnuclear densities deduced from nuclear masses
}

\author{
Kazuhiro Oyamatsu ${ }^{1,2}$ and Kei Iida ${ }^{2,3}$ \\ ${ }^{1}$ Department of Media Theories and Production, Aichi Shukutoku University, \\ Nagakute, Nagakute-cho, Aichi-gun, Aichi 480-1197, Japan \\ ${ }^{2}$ RIKEN Nishina Center, RIKEN, Hirosawa, Wako, Saitama 351-0198, Japan \\ ${ }^{3}$ Department of Natural Science, Kochi University, Akebono-cho, Kochi 780-8520, Japan
}

(Dated: June 30, 2018)

\begin{abstract}
We examine how nuclear masses are related to the density dependence of the symmetry energy. Using a macroscopic nuclear model we calculate nuclear masses in a way dependent on the equation of state of asymmetric nuclear matter. We find by comparison with empirical two-proton separation energies that a smaller symmetry energy at subnuclear densities, corresponding to a larger density symmetry coefficient $L$, is favored. This tendency, which is clearly seen for nuclei that are neutronrich, nondeformed, and light, can be understood from the property of the surface symmetry energy in a compressible liquid-drop picture.
\end{abstract}

PACS numbers: 21.65.Ef, 21.10.Dr

\section{INTRODUCTION}

Saturation of the density and binding energy is a fundamental property of atomic nuclei. Due to this property, the nuclear masses can be described well using a liquid-drop approach. Conventionally, in this approach, the nuclear binding energy $E_{B}$ is written as function of mass number $A$ and charge number $Z$ (or neutron number $N$ ) with the Weizsäcker-Bethe mass formula [1]

$$
-E_{B}=E_{\mathrm{vol}}+E_{\mathrm{sym}}+E_{\mathrm{surf}}+E_{\mathrm{Coul}}
$$

where $E_{\mathrm{vol}}=a_{\mathrm{vol}} A$ is the volume energy, $E_{\mathrm{sym}}=a_{\mathrm{sym}}[(N-Z) / A]^{2} A$ is the symmetry energy, $E_{\mathrm{surf}}=a_{\mathrm{surf}} A^{2 / 3}$ is the surface energy, and $E_{\mathrm{Coul}}=a_{\mathrm{Coul}} Z^{2} / A^{1 / 3}$ is the Coulomb energy. The sum $E_{\mathrm{vol}}+E_{\mathrm{sym}}$ corresponds to the saturation energy of uniform nuclear matter. Since the matter in a nucleus constitutes a strongly interacting system, it remains a challenging theoretical problem to understand the nuclear matter equation of state (EOS) through microscopic calculations that utilize a model of the nuclear force duly incorporating low-energy two-nucleon scattering data and properties of very low-mass nuclei [2]. Furthermore, it is not straightforward to empirically clarify the EOS, although constraints on the EOS obtained from nuclear masses and radii (e.g., Refs. [3 $[6]$ ), observables in heavy-ion collision experiments performed at intermediate and relativistic energies (e.g., Refs. 7, 8]), the isoscalar giant monopole resonance in nuclei (e.g., Ref. [9]), and even X-ray observations of isolated neutron stars [10] and quiescent low-mass X-ray binaries [1] do exist. In this work we will consider such constraints provided by masses of unstable nuclei.

The energy density of bulk nuclear matter is a function of nucleon density $n$ and proton fraction $x$, which are related to the neutron and proton number densities $n_{n}$ and $n_{p}$ as $n_{n}=n(1-x)$ and $n_{p}=n x$. We can generally express the energy per nucleon near the saturation point of symmetric nuclear matter as [12]

$$
w=w_{0}+\frac{K_{0}}{18 n_{0}^{2}}\left(n-n_{0}\right)^{2}+\left[S_{0}+\frac{L}{3 n_{0}}\left(n-n_{0}\right)\right] \alpha^{2} .
$$

Here $w_{0}, n_{0}$ and $K_{0}$ are the saturation energy, the saturation density and the incompressibility of symmetric nuclear matter, and $\alpha=1-2 x$ is the neutron excess. $L$ and $S_{0}$ are associated with the density dependent symmetry energy coefficient $S(n): S_{0}$ is the symmetry energy coefficient at $n=n_{0}$, and $L=3 n_{0}(d S / d n)_{n=n_{0}}$ is the symmetry energy density derivative coefficient (hereafter referred to as the "density symmetry coefficient"). As the neutron excess increases from zero, the saturation point moves in the density versus energy plane. This movement is determined mainly by the parameters $L$ and $S_{0}$. Up to second order in $\alpha$, the saturation energy $w_{s}$ and density $n_{s}$ are given by

$$
w_{s}=w_{0}+S_{0} \alpha^{2}
$$

and

$$
n_{s}=n_{0}-\frac{3 n_{0} L}{K_{0}} \alpha^{2} .
$$

The slope, $y$, of the saturation line near $\alpha=0(x=1 / 2)$ is thus expressed as

$$
y=-\frac{K_{0} S_{0}}{3 n_{0} L} .
$$


In our earlier investigations [6] we explored a systematic way of extracting $L$ and $S_{0}$ from empirical masses and radii of nuclei, together with the parameters, $n_{0}, w_{0}$ and $K_{0}$, characterizing the saturation of symmetric nuclear matter. We first gave an expression for the energy of uniform nuclear matter, which reduces to the phenomenological form (2) in the simultaneous limit $n \rightarrow n_{0}$ and $\alpha \rightarrow 0(x \rightarrow 1 / 2)$. Using this energy expression within a simplified version of the extended Thomas-Fermi approximation, which permits us to determine the macroscopic features of the nuclear ground state, we calculated charges, charge radii and masses of $\beta$-stable nuclei for fixed $A$. Comparing these calculations with empirical values allows us to derive the optimal parameter set for fixed values of the slope $y$ and the incompressibility $K_{0}$. We thus found a strong correlation between $L$ and $S_{0}$. The next step was to calculate the root-mean-square (rms) charge and matter radii of more neutron-rich nuclei that are expected to be produced in radioactive ion beam facilities. The results suggest that the density symmetry coefficient $L$ may be constrained by possible systematic data for the matter radii in a manner that is nearly independent of $K_{0}$.

The reason that we concentrated on radii of unstable nuclei rather than their masses in Ref. [6] was that we originally considered the surface and electrostatic properties as well as shell and pairing effects, which play a role in nuclear masses, to obscure the derivation of the EOS parameters. Nevertheless, in the present analysis, we perform a systematic calculation of nuclear masses from the same framework used for our previous calculations of nuclear radii. Since experimental mass data have been accumulated even for unstable nuclei, we can compare the calculations with the existing data, which is a great advantage over the case of nuclear radii. Comparison of the two-proton separation energy $\left(S_{2 p}(Z, N)=E_{B}(Z, N)-E_{B}(Z-2, N)\right)$ implies that a larger $L$, corresponding to smaller symmetry energy at subnuclear densities, is favored. This tendency is most clearly elucidated from the masses of light, nondeformed, and neutron-rich nuclei. This can be understood from a compressible liquid-drop picture of nuclei in terms of the $L$ dependence of the surface symmetry energy, which has some relevance to the neutron skin thickness [13, 14].

In Sec. II we summarize a macroscopic model of nuclei used here. Calculations of nuclear masses and the comparison with empirical data are illustrated in Sec. III. Our conclusions are presented in Sec. IV.

\section{MACROSCOPIC NUCLEAR MODEL}

In this section, we summarize a macroscopic model of nuclei [ [6], which was constructed in such a way as to reproduce the known global properties of stable nuclei and can be used for describing the masses and radii of unstable nuclei in a manner that is dependent on the EOS of nuclear matter.

The bulk energy per nucleon is an essential ingredient of the macroscopic nuclear model. We set this energy as

$$
\begin{aligned}
w= & \frac{3 \hbar^{2}\left(3 \pi^{2}\right)^{2 / 3}}{10 m_{n} n}\left(n_{n}^{5 / 3}+n_{p}^{5 / 3}\right) \\
& +\left(1-\alpha^{2}\right) v_{s}(n) / n+\alpha^{2} v_{n}(n) / n,
\end{aligned}
$$

where

$$
v_{s}=a_{1} n^{2}+\frac{a_{2} n^{3}}{1+a_{3} n}
$$

and

$$
v_{n}=b_{1} n^{2}+\frac{b_{2} n^{3}}{1+b_{3} n}
$$

are the potential energy densities for symmetric nuclear matter and pure neutron matter, $n_{n}$ and $n_{p}$ are the neutron and proton number densities, $n=n_{n}+n_{p}, \alpha=\left(n_{n}-n_{p}\right) / n$ is the neutron excess, and $m_{n}$ is the neutron mass. Expressions (6) -(8) can well reproduce the microscopic calculations of symmetric nuclear matter and pure neutron matter by Friedman and Pandharipande [15] in the variational method. In this method, the isospin dependence of asymmetric matter EOS is shown to be well approximated by Eq. (6) [16]. (Replacement of the proton mass $m_{p}$ by $m_{n}$ in the proton kinetic energy would make only a negligible difference.) For the later purpose of roughly describing the nucleon distribution in a nucleus, we incorporate into the potential energy densities (7) and (8) a low density behavior $\propto n^{2}$ as expected from a contact two-nucleon interaction. A set of expressions (6) (8) is one of the simplest that reduces to the usual form (2) in the limit of $n \rightarrow n_{0}$ and $\alpha \rightarrow 0$. In fact, the parameters $a_{1}, \cdots, b_{3}$ are related to $n_{0}, w_{0}, K_{0}, S_{0}$, and $L$ as

$$
S_{0}=\frac{1}{6}\left(\frac{3 \pi^{2}}{2}\right)^{2 / 3} \frac{\hbar^{2}}{m_{n}} n_{0}^{2 / 3}+\left(b_{1}-a_{1}\right) n_{0}+\left(\frac{b_{2}}{1+b_{3} n_{0}}-\frac{a_{2}}{1+a_{3} n_{0}}\right) n_{0}^{2},
$$




$$
\begin{gathered}
\frac{1}{3} n_{0} L=\frac{1}{9}\left(\frac{3 \pi^{2}}{2}\right)^{2 / 3} \frac{\hbar^{2}}{m_{n}} n_{0}^{5 / 3}+\left(b_{1}-a_{1}\right) n_{0}^{2}+2\left(\frac{b_{2}}{1+b_{3} n_{0}}-\frac{a_{2}}{1+a_{3} n_{0}}\right) n_{0}^{3} \\
-\left[\frac{b_{2} b_{3}}{\left(1+b_{3} n_{0}\right)^{2}}-\frac{a_{2} a_{3}}{\left(1+a_{3} n_{0}\right)^{2}}\right] n_{0}^{4}, \\
w_{0}=\frac{3}{10}\left(\frac{3 \pi^{2}}{2}\right)^{2 / 3} \frac{\hbar^{2}}{m_{n}} n_{0}^{2 / 3}+a_{1} n_{0}+\frac{a_{2} n_{0}^{2}}{1+a_{3} n_{0}}, \\
K_{0}=-\frac{3}{5}\left(\frac{3 \pi^{2}}{2}\right)^{2 / 3} \frac{\hbar^{2}}{m_{n}} n_{0}^{2 / 3}+\frac{18 a_{2} n_{0}^{2}}{\left(1+a_{3} n_{0}\right)^{3}}, \\
0=\frac{1}{5}\left(\frac{3 \pi^{2}}{2}\right)^{2 / 3} \frac{\hbar^{2}}{m_{n}} n_{0}^{-1 / 3}+a_{1}+\frac{2 a_{2} n_{0}}{1+a_{3} n_{0}}-\frac{a_{2} a_{3} n_{0}^{2}}{\left(1+a_{3} n_{0}\right)^{2}} .
\end{gathered}
$$

We determine the parameters $a_{1}, \cdots, b_{3}$ in such a way that the charge number, charge radius, and mass of stable nuclei calculated in a macroscopic nuclear model constructed in Ref. [6] are consistent with the empirical data. In the course of this determination, we fix $b_{3}$, which controls the EOS of matter for large neutron excess and high density, at $1.58632 \mathrm{fm}^{3}$. This value was obtained by one of the authors [17] in such a way as to reproduce the neutron matter energy of Friedman and Pandharipande [15]. Change in this parameter would make no significant difference in the determination of the other parameters and the final results for nuclear masses.

We describe macroscopic nuclear properties in a way dependent on the EOS parameters $a_{1}, \cdots, b_{3}$ by using a Thomas-Fermi model [6]. The essential point of this model is to write down the total energy of a nucleus of mass number $A$ and charge number $Z$ as a function of the density distributions $n_{n}(\mathbf{r})$ and $n_{p}(\mathbf{r})$ in the form

$$
E=E_{b}+E_{g}+E_{C}+N m_{n} c^{2}+Z m_{p} c^{2}
$$

where

$$
E_{b}=\int d^{3} r n(\mathbf{r}) w\left(n_{n}(\mathbf{r}), n_{p}(\mathbf{r})\right)
$$

is the bulk energy,

$$
E_{g}=F_{0} \int d^{3} r|\nabla n(\mathbf{r})|^{2}
$$

is the gradient energy with adjustable constant $F_{0}$,

$$
E_{C}=\frac{e^{2}}{2} \int d^{3} r \int d^{3} r^{\prime} \frac{n_{p}(\mathbf{r}) n_{p}\left(\mathbf{r}^{\prime}\right)}{\left|\mathbf{r}-\mathbf{r}^{\prime}\right|}
$$

is the Coulomb energy, and $N=A-Z$ is the neutron number. This functional allows us to connect the EOS and the nuclear binding energy through the bulk energy part $E_{b}$. For simplicity we use the following parametrization for the nucleon distributions $n_{i}(r)(i=n, p)$ :

$$
n_{i}(r)= \begin{cases}n_{i}^{\mathrm{in}}\left[1-\left(\frac{r}{R_{i}}\right)^{t_{i}}\right]^{3}, & r<R_{i}, \\ 0, & r \geq R_{i} .\end{cases}
$$

This parametrization allows for the central density, half-density radius, and surface diffuseness for neutrons and protons separately.

In order to construct the nuclear model in such a way as to reproduce empirical masses and radii of stable nuclei, we first extremized the binding energy with respect to the particle distributions for fixed mass number, five EOS parameters $a_{1}, \cdots, b_{2}$, and gradient coefficient $F_{0}$. Next, for various sets of the incompressibility $K_{0}$ and the density symmetry coefficient $L$, we obtained the remaining three EOS parameters $n_{0}, w_{0}$, and $S_{0}$ and the gradient coefficient 


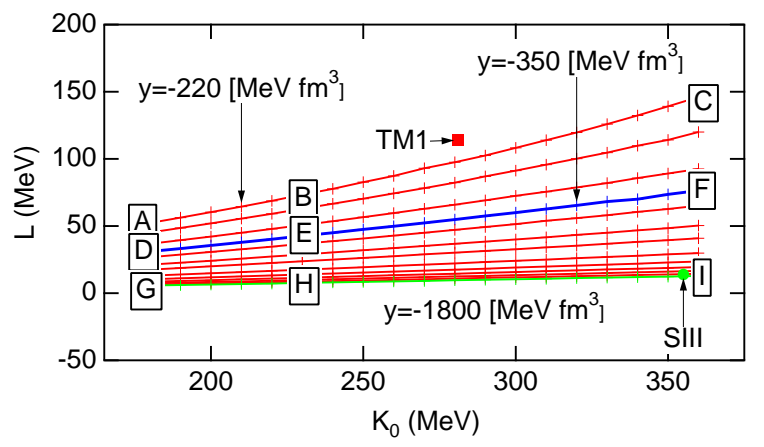

FIG. 1: (Color online) The sets of $\left(L, K_{0}\right)$ (crosses) consistent with the mass and radius data for stable nuclei. The thin lines are lines of constant $y$. The labels A-I denote the sets for which we performed detailed calculations of the ground state properties of inhomogeneous nuclear matter at subnuclear densities [18]. In this paper we often focus on the EOS models with the parameter sets $\mathrm{C}$ and G. For comparison, the values calculated from two mean-field models [TM1 (square) and SIII (dot)], which are known to be extreme cases [3], are plotted. The plot shows that our sets of $\left(L, K_{0}\right)$ effectively cover such extreme cases and constraints on $L$ and $K_{0}$ from other observables [19].

$F_{0}$ by fitting the calculated optimal values of charge number, mass excess, rms charge radius to empirical data for stable nuclei on the smoothed $\beta$ stability line [17] and by using Eqs. (91)-(13). In the range of the parameters $0<L<160$ $\mathrm{MeV}$ and $180 \mathrm{MeV}<K_{0}<360 \mathrm{MeV}$, as long as $y \lesssim-200 \mathrm{MeV} \mathrm{fm}^{3}$, we obtained a reasonable fitting to such data (see Fig. 1). As a result of this fitting, the parameters $n_{0}, w_{0}, S_{0}$, and $F_{0}$ are constrained as $n_{0}=0.14-0.17 \mathrm{fm}^{-3}$, $w_{0}=-16 \pm 1 \mathrm{MeV}, S_{0}=25-40 \mathrm{MeV}$, and $F_{0}=66 \pm 6 \mathrm{MeV} \mathrm{fm}^{5}$. We remark that a negative $L$ is inconsistent with the fact that the size of $A=17,20,31$ isobars deduced from the experimental values of the interaction cross section tends to increase with neutron/proton excess [20]. This inconsistency can be seen from Eq. (4) which shows that the saturation density $n_{s}$ increases (and hence the isobar size decreases) with neutron/proton excess for a negative $L$. We also note that the fitting gives rise to a relation nearly independent of $K_{0}$,

$$
S_{0} \approx B+C L,
$$

where $B \approx 28 \mathrm{MeV}$ and $C \approx 0.075$. As we shall see, this linear relation plays a part in the $L$ dependence of calculated masses at large neutron excess.

We remark that in the range of the EOS parameters $L$ and $K_{0}$ shown in Fig. 1, the calculations agree well with a more extended data set of nuclear masses for $A \geq 2$ [21] and charge radii for $A \geq 50$ [22]. The rms deviations of the calculated masses from the measured values are $\sim 3-5 \mathrm{MeV}$, which are comparable with the deviations obtained from a Weizsäcker-Bethe formula, while the rms deviations of the calculated charge radii from the measured values are about $0.06 \mathrm{fm}$, which are comparable with the deviations obtained from the $A^{1 / 3}$ law. As we shall see, detailed comparison with empirical masses in terms of two-proton separation energy allows us to tell which of the EOS models with large $L$ and with small $L$ are more favored.

\section{NUCLEAR MASSES}

We now proceed to evaluate nuclear masses from various EOS models with the parameter set $\left(L, K_{0}\right)$ consistent with the mass and radius data for stable nuclei by minimizing Eq. (14) for fixed $N$ and $Z$. The results are then compared with the existing data in terms of the two-proton separation energy. We finally discuss the $L$ dependence of the calculated masses within the framework of a compressible liquid-drop picture of nuclei.

We begin by illustrating the calculated and experimental values of the two-proton separation energy $S_{2 p}$ for $\mathrm{O}, \mathrm{Mg}$, $\mathrm{Ca}, \mathrm{Ni}, \mathrm{Sn}$, and $\mathrm{Pb}$ isotopes. The two-proton separation energy is useful partly because the even-odd staggering is essentially cancelled out and partly because the isotope dependence except for shell gaps is smooth according to the Yamada-Matumoto systematics [23]. In fact, as can be seen from Fig. 2 in which the two-proton separation energy minus the one calculated from a Weizsäcker-Bethe mass formula (11) with the coefficients $a_{\mathrm{vol}}=-15.5391 \mathrm{MeV}$, $a_{\text {sym }}=22.7739 \mathrm{MeV}, a_{\text {surf }}=16.9666 \mathrm{MeV}$, and $a_{\text {Coul }}=0.703893 \mathrm{MeV}$ [24], i.e., $S_{2 p}-S_{2 p}^{\mathrm{WB}}$, is plotted, the empirical values behave monotonously with neutron excess except for the vicinity of $N=Z$ and neutron magic numbers and for the deformed region. Note that this monotonous behavior is clearer for $\mathrm{O}, \mathrm{Mg}$, and $\mathrm{Ca}$ than for $\mathrm{Ni}, \mathrm{Sn}, \mathrm{and} \mathrm{Pb}$.

We remark that the macroscopic nuclear model summarized in the previous section was originally used for systematic calculations of charge and matter radii of nuclei of $A \gtrsim 50$ [6]. Here, we performed systematic calculations of masses 

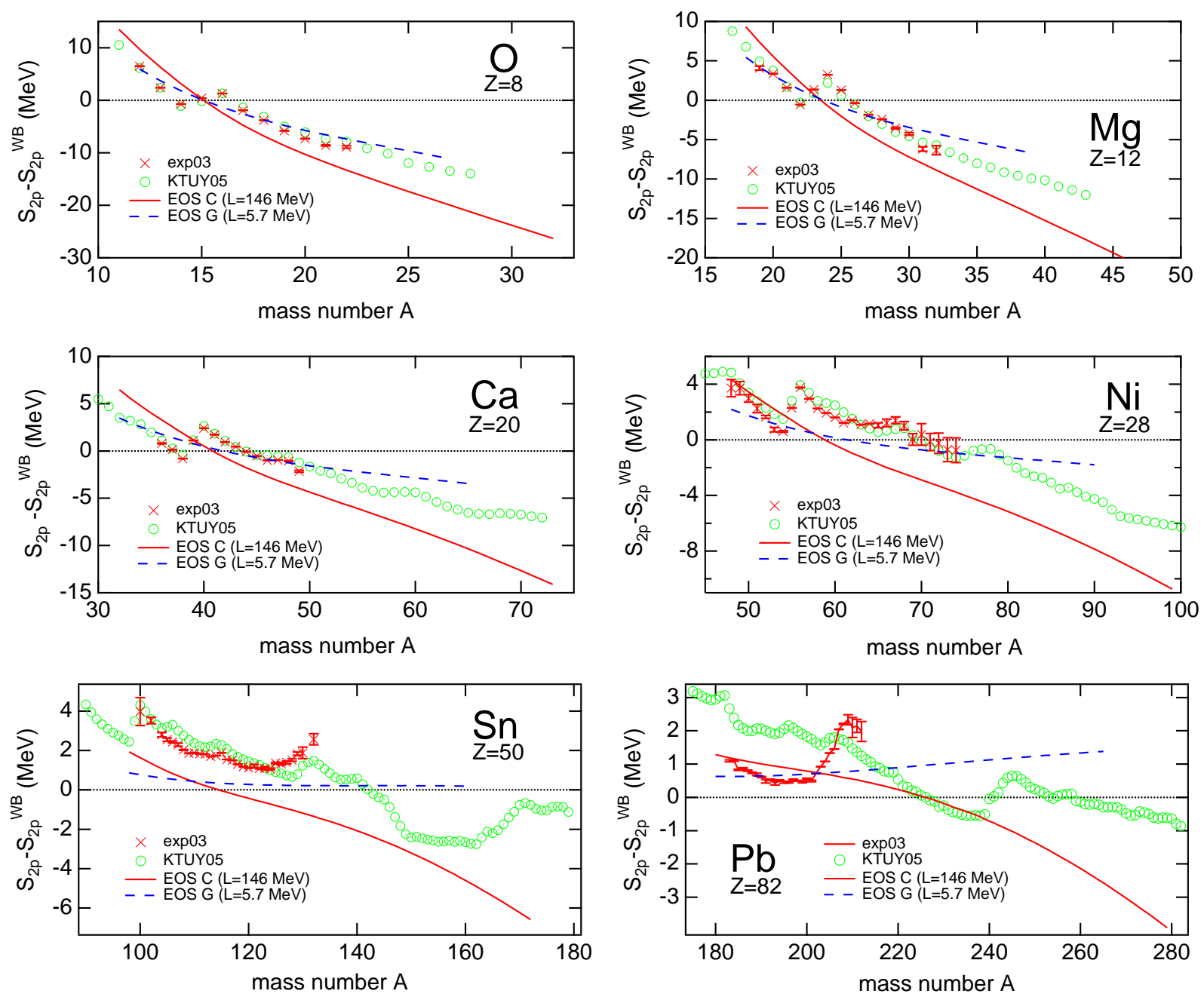

FIG. 2: (Color online) The two-proton separation energy, having the one calculated from a Weizsäcker-Bethe mass formula 24 subtracted out, for $\mathrm{O}, \mathrm{Mg}, \mathrm{Ca}, \mathrm{Ni}, \mathrm{Sn}$, and $\mathrm{Pb}$ isotopes. The empirical values [21], the calculated values from the EOS models $\mathrm{G}$ and $\mathrm{C}$, and the values obtained from a contemporary mass formula [25] are plotted in each panel.

of nuclei including $\mathrm{O}, \mathrm{Mg}$, and Ca isotopes. Applicability to lighter nuclei can be confirmed from Fig. 2, which shows that differences between the calculated and empirical values of $S_{2 p}$ for stable nuclei are limited within $3 \mathrm{MeV}$.

The calculated values of $S_{2 p}$, neither including deformation, the Wigner term, nor shell corrections, show a smooth dependence on neutron excess in a way different between the EOS models $\mathrm{G}(L=5.7 \mathrm{MeV})$ and $\mathrm{C}(L=146 \mathrm{MeV})$. (The Weizsäcker-Bethe formula, which is based on the incompressible liquid-drop model for nuclei, is independent of the parameters $L$ and $K_{0}$ characterizing the density dependence of the EOS.) In order to examine how the calculated values of $S_{2 p}$ depend on $L$ and $K_{0}$, we plot in Fig. 3 the results obtained for ${ }^{78} \mathrm{Ni}$ and ${ }^{22} \mathrm{O}$ from the EOS models with various values of $L$ and $K_{0}$ shown in Fig. 1 . The results decrease with $L$ almost linearly, while they are nearly independent of $K_{0}$. Accordingly, for various values of $L$ one can predict where $S_{2 p}-S_{2 p}^{\mathrm{WB}}$ is located in Fig. 2 by interpolation or extrapolation of the results from the EOS models G and C. As far as the slope of $S_{2 p}-S_{2 p}^{\mathrm{WB}}$ is concerned, the result with the EOS model C seems to be more consistent with the empirical behavior, particularly for light nuclei.

We also notice from Fig. 2 that there is a roughly uniform offset between the empirical values and the values calculated from the EOS model $\mathrm{C}$ at $N>Z$. This offset comes mainly from the proton shell gaps. In fact, it is effectively cancelled out in a region away from $Z=N$ and neutron magic numbers by taking a difference, $S_{2 p}(Z, N)-$ $S_{2 p}(Z, N-2) \equiv 4 \delta V_{n p}$, as can be seen from Fig. 4. In contrast, the empirical behavior of this difference shows a $N=Z$ and neutron shell structure in a more exaggerated manner. As discussed in Ref. 26], the smoothed behavior of $\delta V_{n p}$ is related to the coefficients $a_{\mathrm{sym}}$ and $a_{\mathrm{ssym}}$ affixed to $A[(N-Z) / A]^{2}$ and $A^{2 / 3}[(N-Z) / A]^{2}$ in the mass 

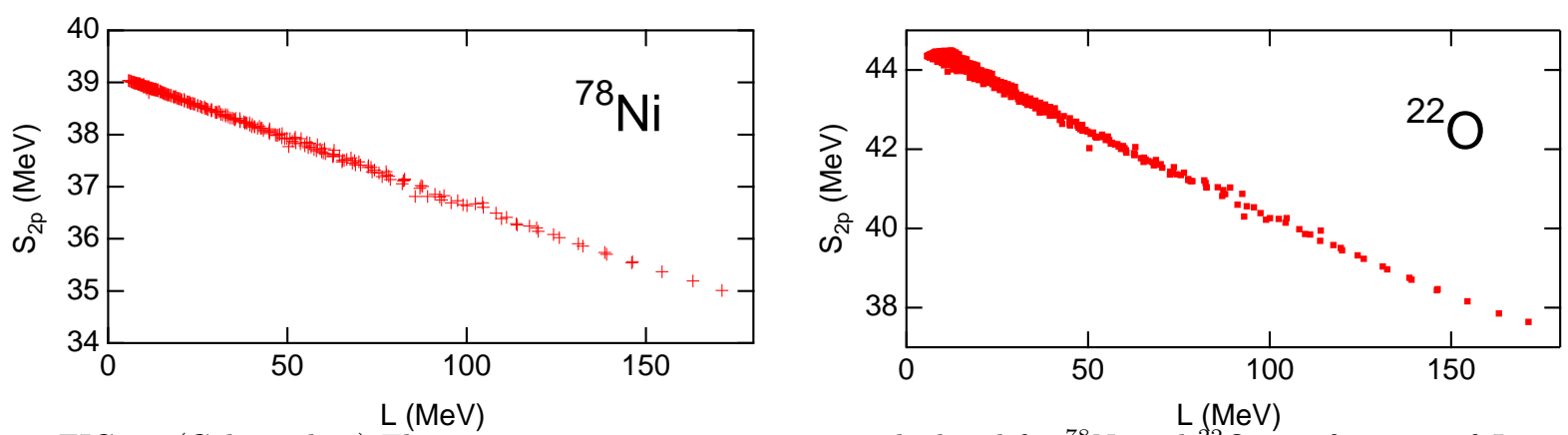

FIG. 3: (Color online) The two-proton separation energy calculated for ${ }^{78} \mathrm{Ni}$ and ${ }^{22} \mathrm{O}$ as a function of $L$.
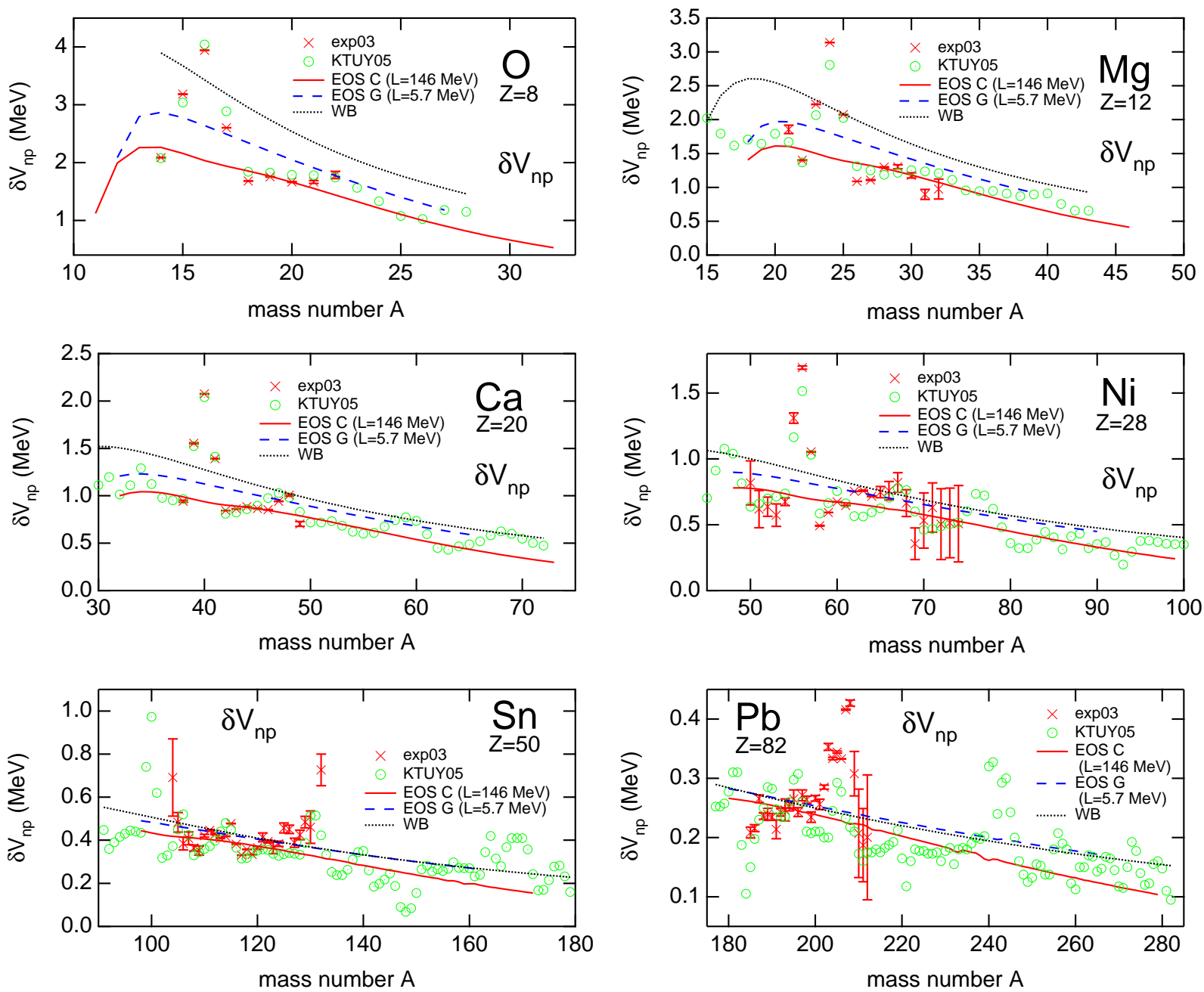

FIG. 4: (Color online) $\delta V_{n p}$ for $\mathrm{O}, \mathrm{Mg}, \mathrm{Ca}, \mathrm{Ni}, \mathrm{Sn}$, and $\mathrm{Pb}$ isotopes. In each panel, the empirical values [21], the values from a contemporary mass formula [25] and a Weizsäcker-Bethe mass formula [24], and the calculated values from the EOS models C and $\mathrm{G}$ are plotted.

formula (1) (with the surface symmetry term added) by

$$
\delta V_{n p} \approx 2\left(a_{\mathrm{sym}}+a_{\mathrm{ssym}} A^{-1 / 3}\right) / A .
$$

Our results for $\delta V_{n p}$ suggest that the $L$ dependence lies in the parameters $a_{\text {sym }}$ and $a_{\text {ssym }}$. In fact, this suggestion can be understood within the framework of a compressible liquid-drop model in which nuclei in equilibrium are allowed to have a density different from the saturation density $n_{0}$ of symmetric nuclear matter. If one ignores Coulomb and 

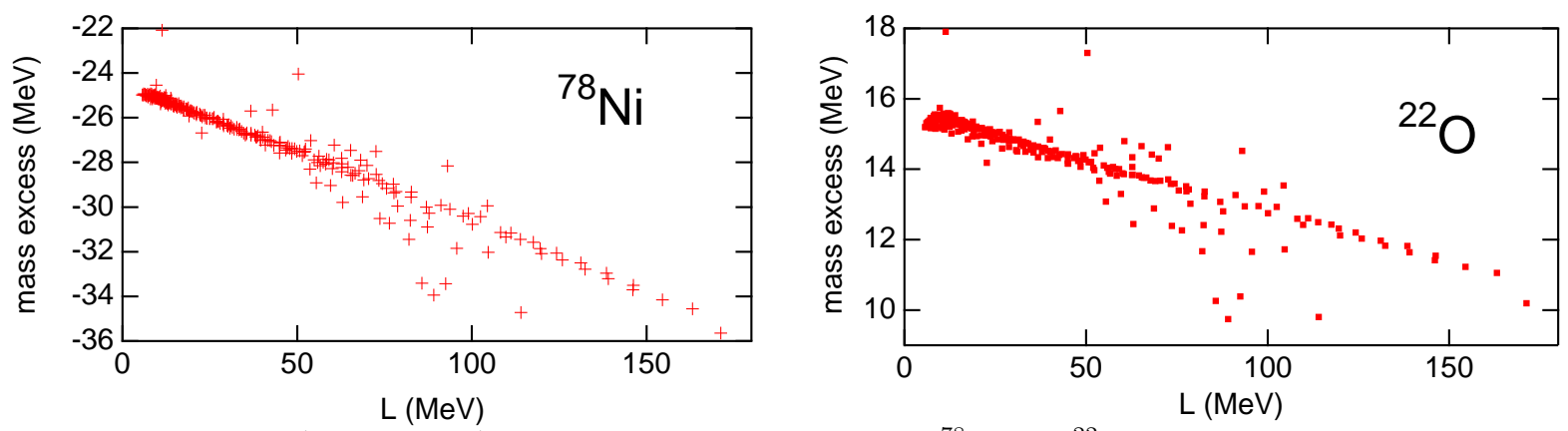

FIG. 5: (Color online) The mass excess calculated for ${ }^{78} \mathrm{Ni}$ and ${ }^{22} \mathrm{O}$ as a function of $L$.

surface corrections, the equilibrium density and energy per nucleon of a liquid-drop correspond to $n_{s}$ and $w_{s}$, which are given by Eqs. (4) and (3) for nearly symmetric nuclear matter. Note that the parameter $a_{\text {sym }}$ is characterized by the symmetry energy coefficient $S_{0}$, which in turn is related to $L$ by Eq. (19). Since one obtains a larger $a_{\text {sym }}$ for larger $L$, the effect of $a_{\mathrm{sym}}$ tends to increase $\delta V_{n p}$ with $L$, which is in the wrong direction. This effect, therefore, has to be dominated by the effect of $a_{\text {ssym }}$. This is consistent with the fact that the $L$ dependence of the calculated $S_{2 p}$ is clearer for lighter nuclei and also with the tendency that the calculated mass decreases with $L$ for neutron-rich nuclei (see Fig. 5).

The effect of $a_{\text {ssym }}$ can be understood by considering the density-dependent surface tension [13],

$$
\sigma\left(n_{\mathrm{in}}, \alpha_{\mathrm{in}}\right)=\sigma_{0}\left[1-C_{\mathrm{sym}} \alpha_{\mathrm{in}}^{2}+\chi\left(\frac{n_{\mathrm{in}}-n_{0}}{n_{0}}\right)\right],
$$

where $n_{\text {in }}$ and $\alpha_{\text {in }}$ are the density and neutron excess inside a liquid-drop, $\sigma_{0}=\sigma\left(n_{0}, 0\right), C_{\text {sym }}$ is the surface symmetry energy coefficient, and $\chi=\left(n_{0} / \sigma_{0}\right) \partial \sigma /\left.\partial n_{\text {in }}\right|_{n_{\text {in }}=n_{0}, \alpha_{\text {in }}=0}$. By substituting $n_{s}$ into $n_{\text {in }}$, one obtains

$$
\sigma\left(n_{s}, \alpha_{\mathrm{in}}\right)=\sigma_{0}\left[1-\left(C_{\mathrm{sym}}+\frac{3 L \chi}{K_{0}}\right) \alpha_{\mathrm{in}}^{2}\right] .
$$

Thus, $a_{\mathrm{ssym}} A^{2 / 3}$ behaves as $-4 \pi \sigma_{0} R^{2}\left(C_{\mathrm{sym}}+\frac{3 L \chi}{K_{0}}\right)$, with the liquid-drop radius $R$. The condition $\chi>0$, which is suggested by various nuclear models including the macroscopic one utilized here [27], is desirable for understanding of the $L$ dependence of $\delta V_{n p}$. We remark that this condition is suggested by various mean-field models because the calculated neutron skin thickness, which is basically proportional to $-a_{\text {ssym }}$, increases with $L[14]$.

\section{CONCLUSIONS}

We have analyzed the influence of the density dependence of the symmetry energy on nuclear masses by using a macroscopic nuclear model that depends explicitly on the EOS of nuclear matter. We find that the $L$ dependence of the calculated masses comes mainly from the surface property through the density and neutron excess dependence of the surface tension.

In making reasonable estimates of $L$ from empirical masses, we find that empirical two-proton separation energies for neutron-rich, light nuclei are useful, implying a larger $L$ value. However, we still have two caveats. First, Eq. (22) suggests that uncertainties in $\chi / K_{0}$ affect the prediction of the value $L$. Second, a smooth isotope dependence of empirical mass data is hard to derive. Consequently, such estimates of $L$ are closely connected to determination of the parameters $\chi$ and $K_{0}$ characterizing the density dependence of the surface and bulk energy and to understanding of the discrete behavior of $S_{2 p}$ and $\delta V_{n p}[26,28,29]$.

\section{Acknowledgments}

We are grateful to Drs. H. Koura and A. Kohama for useful discussion on this and related subjects. We acknowledge the hospitality of the Yukawa Institute for Theoretical Physics during the workshop "New Frontiers in QCD 2010," 
where this work was finished.

[1] J.M. Blatt and V.F. Weisskopf, Theoretical Nuclear Physics (Wiley, New York, 1952).

[2] H. Heiselberg and V.R. Pandharipande, Ann. Rev. Nucl. Part. Sci. 50, 481 (2000).

[3] K. Oyamatsu, I. Tanihata, Y. Sugahara, K. Sumiyoshi, and H. Toki, Nucl. Phys. A634, 3 (1998).

[4] B.A. Brown, Phys. Rev. Lett. 85, 5296 (2000).

[5] K.C. Chung, C.S. Wang, and A.J. Santiago, nucl-th/0102017

[6] K. Oyamatsu and K. Iida, Prog. Theor. Phys. 109, 631 (2003).

[7] P. Danielewicz, R. Lacey, and W.G. Lynch, Sci. 298, 1592 (2002).

[8] B.A. Li, L.W. Chen, and C.M. Ko, Phys. Rep. 464, 113 (2008).

[9] D.H. Youngblood, H.L. Clark, and Y.-W. Lui, Phys. Rev. Lett. 82, 691 (1999).

[10] J.M. Lattimer and M. Prakash, Sci. 304, 536 (2004).

[11] S. Guillot, R.E. Rutledge, L. Bildsten, E.F. Brown, G.G. Pavlov, and V.E. Zavlin, Mon. Not. R. Astron. Soc. 392, 665 (2009).

[12] J.M. Lattimer, Ann. Rev. Nucl. Part. Sci. 31, 337 (1981).

[13] K. Iida and K. Oyamatsu, Phys. Rev. C 69, 037301 (2004).

[14] M. Warda, X. Viñas, X. Roca-Maza, and M. Centelles, Phys. Rev. C 80, 024316 (2009).

[15] B. Friedman and V.R. Pandharipande, Nucl. Phys. A361, 502 (1981).

[16] I.E. Lagaris and V.R. Pandharipande, Nucl. Phys. A369, 470 (1981).

[17] K. Oyamatsu, Nucl. Phy. A561, 431 (1993).

[18] K. Oyamatsu and K. Iida, Phys. Rev. C 75, 015801 (2007).

[19] See, e.g., Fig. 6 in Ref. [14]; G. Colò, N. Van Giai, J. Meyer, K. Bennaceur, and P. Bonche, Phys. Rev. C 70, 024307 (2004).

[20] A. Ozawa et al., Phys. Lett. B344, 18 (1994); L. Chulkov et al., Nucl. Phys. A603, 219 (1996); A. Ozawa et al., Nucl. Phys. A709, 60 (2002).

[21] G. Audi, A.H. Wapstra, and C. Thibault, Nucl. Phys. A729, 337 (2003).

[22] H. de Vries, C.W. de Jager, and C. de Vries, At. Data Nucl. Data Tables 36, 495 (1987).

[23] M. Yamada and Z. Matumoto, J. Phys. Soc. Jpn. 16, 1497 (1961).

[24] M. Yamada, Prog. Theor. Phys. 32, 512 (1964).

[25] H. Koura, T. Tachibana, M. Uno, and M. Yamada, Prog. Theor. Phys. 113, 305 (2005).

[26] M. Stoitsov, R.B. Cakirli, R.F. Casten, W. Nazarewicz, and W. Satuła, Phys. Rev. Lett. 98, 132502 (2007).

[27] For example, the Fermi gas model gives $\chi=4 / 3$.

[28] L. Chen et al., Phys. Rev. Lett. 102, 122503 (2009).

[29] A. Gelberg, H. Sakurai, M.W. Kirson, and S. Heinze, Phys. Rev. C 80, 024307 (2009). 